\title{
IMPACT OF THE SURFACE ROUGHNESS DESCRIPTION ON THE ELECTRICAL CONTACT RESISTANCE OF OHMIC SWITCHES UNDER LOW ACTUATION FORCES
}

\author{
Fabienne Pennec, David Peyrou, Dimitri Leray, Patrick Pons, Robert Plana and Frédéric Courtade
}

\begin{abstract}
At the present time the insertion of RF MEMS switches into real architecture requires reduced actuation voltage, reduced dimensions and a better control of the electrical and electromechanical behavior that gives more importance to surface effects, their understanding and modeling. The use of such devices needs the development of methods for estimating the contact performances as a function of surface roughness, contact materials and contact topologies. With the increase of computation capabilities the rough surface topography can be implemented in the finite element model but implies long calculation times or even calculation overloading if a high definition of the roughness is desired. To reduce these limitations, assumptions on the micro-geometry are required. This work treats, by use of finite element modeling, the influence of the definition of roughness of contacting switch members on the electrical contact resistance of resistive switches and investigates the error introduced by using a minimal defined AFM sampling interval of $10 \mathrm{~nm}$. The present numerical analysis is implemented for switch test structures.
\end{abstract}

Index Terms - ANSYS11, deterministic, discretization step, electrical contact resistance, finite element method, metal-tometal contact, RF MEMS switches, roughness.

NOMENCLATURE

\begin{tabular}{|c|c|}
\hline$a$ & Microcontact spot radius, nm. \\
\hline$a_{\text {eff }}$ & Effective contact spot radius, $\mathrm{nm}$. \\
\hline$l_{e}$ & electron mean free path, nm. \\
\hline$K$ & Knudsen number where $K=\frac{l_{e}}{a_{e f f}}$. \\
\hline$\Gamma(\mathrm{K})$ & $\begin{array}{l}\text { slowly varying Gamma function of unity order } \\
\Gamma(K)=\frac{2}{\pi} \int_{0}^{\infty} e^{-K x} \operatorname{Sinc}(x) d x\end{array}$ \\
\hline$R_{G B}$ & $\begin{array}{l}\text { Electrical contact resistance in ballistic transport or } \\
\text { Sharvin's resistance, } \Omega \text {. }\end{array}$ \\
\hline$R_{S D}$ & $\begin{array}{l}\text { Electrical contact resistance in diffusive transport or } \\
\text { Holm's resistance, } \Omega \text {. }\end{array}$ \\
\hline$R_{W}$ & Wexler resistance in quasi-ballistic transport, $\Omega$. \\
\hline$\rho$ & electrical resistivity, $\mathrm{n} \Omega . \mathrm{m}$. \\
\hline$r_{a}$ & Average roughness, nm. \\
\hline & Standard deviation, nm. \\
\hline
\end{tabular}

Manuscript received November 18, 2009. This work was supported in part by the Laboratory for Analysis and Architecture of Systems (LAAS) and the Centre National d'Etudes Spatiales (CNES).
F. Pennec, D. Peyrou, , P. Pons and R. Plana are with LAAS - CNRS Toulouse University - 7 av. du Colonel Roche - 31077 Toulouse cedex 4 France (e-mail: fpennec@laas.fr)

D. Leray is with the INSA of Toulouse, a School of Engineering in 5 years, under the authority of the Ministry of Education and Research, 135, avenue de Rangueil - 31077 Toulouse Cedex 4 - France

F. Courtade is with the Centre National d'études Spatiales (CNES), Centre spatial de Toulouse - 18, avenue Edouard Belin - 31401 Toulouse cedex 9 - France

\section{INTRODUCTION}

With their extremely low mass and volume, low power consumption and easy integration with electronics, RF MEMS switches have a strong potential to reduce the size and mass of satellite systems and spacecraft with significant cost reduction [1]. Nevertheless, these devices with mobile structures still have some issues to be resolved before they can be successfully integrated into industrial products. The first issue deals with the actuation medium and the corresponding reliability. For the ohmic RF MEMS switches, it has been identified that most of the limitations are related to the quality and the repeatability of the contact that drive the RF performance (insertion losses, insulation, power handling) and the reliability [2]. In fact, surface roughness has many effects in contacting electrical connectors. RF MEMS switches display a roughness at nanometer scale. As contact forces in such devices are in the order of a few tens of micro-newtons to ten millinewtons [3-5], only a few asperities on the contact surfaces are effectively in contact when rough surfaces are pressed against each other. The roughness associated with surfaces yields an actual contact area equal to a fraction of the apparent or nominal contact area [6-9]. In classical contacts, generated forces are strong enough to neglect the surface effects. By contrast quality and reliability of microswitch contact is determined by the presence of these nanoasperities. In order to propose new generation of RF MEMS devices, it is important to get a deeper insight on the physics of contact in order to choose appropriate materials, topology and architecture.

Many methodologies are available to reach this goal but can include major drawbacks. An experimental method is notably exposed to many difficulties, concerning the fabrication technology or the experimental measurements. The testing and development of contact material or contact 
topology can be addressed with a dedicated experimental set up for monitoring test structures. Nevertheless, it is difficult to perform the tests under realistic conditions and in particular to duplicate the switch geometry, the contact geometry and the contact force. Moreover, the fabrication process must be optimized and it may take many months to fabricate a set of switches to test a single candidate contact material or contact bump shape. Finally, this method is costly whereas the results interpretation can prove to be difficult when many physical phenomena appear together (heating, creep, surface contamination...).

About the theoretical models, many assumptions and simplifications are involved. They ignore some or all of the correlation between asperities, implying that asperities are far apart. Moreover, bulk deformation is neglected and plasticity models at the asperities do not consider large deformation theory.

With the increase of computation capabilities, the numerical methods are of great interest. For an elasticplastic material, finite element analysis is well-adapted and is robust enough to consider interaction between asperities as well as bulk deformation. A numerical method using finite elements code is thus selected to describe the roughness of contact surface and perform the mechanical contact study.

To perform this study, the determination of the real contact area is crucial since it allows the electric current to flow and affects the electrical contact resistance value. A precise description of the actual surface topographies to determine the shape or the height distribution of asperities could improve the forecasting of real contact area. This task is the major difficulty for modeling the contact between rough surfaces.

In this work, we analyzed the more complied approach for the description of the rough surface of micro-switches. We concentrate our research efforts on the choice of a scan size and a discretization step limit of the roughness. The goal is to make the contact analysis using a finite element approach without losing information on the complex micro-geometry and interactions between asperities.

The model is implemented on test structures. Finite element contact analysis are thus carried out including the actual topography of contact surfaces with a minimal defined AFM sampling interval of 10nm.

\section{METHODOLOGY FOR ROUGHNESS MEASUREMENT}

\section{A. Roughness measurement on typical surfaces}

Historically three different approaches have been developed to describe the surfaces: a statistical approach, based on a stochastic analysis, a fractal approach using scale-independent fractal parameters and a deterministic one involving the actual surfaces topography $[10,11]$.

The contact surfaces of devices that are produced by standard technological process are of random nature and can thus be described by a stochastic approach. This approach requires the definition of heights distribution functions and some assumptions on asperities shape.
Greenwood and Williamson [12] have developed the most widely used statistical model. They use the hypothesis that the rough surface is composed of spherical asperities having identical radii of curvature and heights following a Gauss distribution about a mean plane. The model allows us to evaluate the effective contact area and maximal contact pressure of a rough contact. The statistical approach requires a few parameters and is simple to carry out when their determination is immediate. Stochastic models have thus often been preferred to reduce computational times since they refer to a few parameters [12]. Nevertheless the choice of statistical parameters can be conflicting. The roughness measurement consists indeed of signal processing on surface profiles and the measurement data can be interpreted in different manners. Statistical roughness parameters are also strongly dependent on the resolution of the measuring instrument.

Another solution is to use a fractal approach to study the irregular texture of rough contact surfaces. The fractal theory has been highlighted by the mathematician Benoît Mandelbrot. He pointed out the connection between surface roughness and fractal dimension considering the multiscale nature of surface topography that is not accounted for by the statistical models. In this way, random surface texture is characterized by scale independent fractal parameters. A few authors applied finite element method to the fractal surface $[13,14]$.

When the distribution of peaks and valleys is not random on the rough surface but deterministic, we use in preference a deterministic approach. Actual heights on the rough surface are captured at discrete points with a measuring instrument and are thus strongly dependent on its resolution. The deterministic approach has the great interest to avoid making assumptions on heights distribution and asperities shape. Moreover the multi-scale nature of the rough surfaces can be taken into account if topographies are captured with a sampling interval small enough to include the finest details. However a deterministic approach can imply a tremendous amount of data if it is selected.

In any case an experimental measurement of the surface topography is required to determine statistical and fractals parameters or actual heights of the surface at discrete points.

Depending on the particular application, a roughness description method and a measuring instrument can be chosen.

\section{B. Surface roughness description under low forces}

The choice of a suitable instrument capable to describe the finest details of the rough surfaces involved in the contact of both parts of the micro-switch is primordial [15]. Electrical contact of such devices is generally strongly localized due to the patterning of contact bump under the suspended membrane or directly on the coplanar line. With the typical contact force generated by the device $(<150 \mu \mathrm{N})$, the apparent contact radius is not larger than $250 \mathrm{~nm}$. Since the contacting surface dimensions are often less than 500 
nm along one direction, we need an instrument which can measure high frequency roughness. The AFM instrument is well adapted since its sampling interval limit depends on scan size and on the number of data points registered for the sample. For example, an AFM measurement with a scan size of $500 \times 500 \mathrm{~nm}^{2}$ and containing $512 \times 512$ data points will give a sampling interval of about $1 \mathrm{~nm}$. The choice of the sampling interval is crucial and has to be of nanometer order as contact first occurs on the top of nanoasperities, which deform under the action of the applied load in a predominantly plastic manner. According to Bushan et al. [16], as the load is increased, nano-asperities deform readily and merge in larger micro-asperities with subsequent deformation being elastic or plastic.

Microelectronic thin layer deposition (sputtering, evaporating, electroplating) can be in general characterized by a random surface topography and thus can be described statistically if appropriate asperity distribution functions are chosen or with fractals if appropriate fractal parameters are selected. However for the dimensions of examined surface in a contact of an ohmic micro-switch the random texture is not obvious. Figure 1 shows the topographies, captured by AFM, of the membrane surface and of the signal line with sample dimensions of $500 \times 500 \mathrm{~nm}^{2}$. Whereas the contact surface of the membrane seems to be random, the contact surface of the line appears to be disturbed by asperities higher than the neighborhood. These observations suggest that these samples have undulations larger than 500nm. It is obvious that the contact between both surfaces will occur through the higher asperities. The estimation of statistical parameters for this sample size is no longer feasible. And thus a deterministic description of the lower contact surface is required.

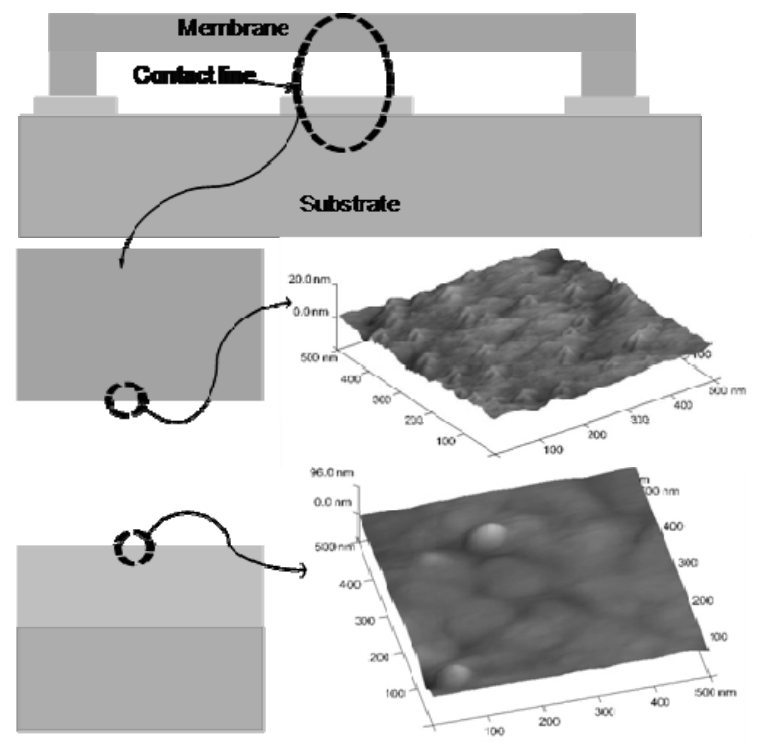

Fig. 1. Contact surface topographies of the resistive micro-switches captured by AFM (membrane surface: $R_{a}=1,14 \mathrm{~nm}, \mathrm{R}_{\mathrm{q}}=1,43 \mathrm{~nm}$; signal line: $\left.R_{a}=3,24 n m, R_{q}=4,53 n m\right)$.

It has to be added that the surface roughness of contact materials will depend on the switching history of the device. A surface which appears initially stochastic will be of deterministic nature as soon as a few repeated cycles are initiated. In fact exploring the domain of very low contact forces requires high attention to surface cleanness. A method often used for cleaning metallic surfaces from contaminating films is called 'Shaltreinigung' and consists of performing some switching cycles under electric load before all measurements [4]. To be closer to the reality, the described rough surfaces have to be thus the altered contact surfaces of the resistive micro-switches.

The use of a deterministic approach is more appropriate in our case. To get accurate results, a study on a certain amount of samples is required.

The deterministic method includes unfortunately some difficulties of an experimental nature and other difficulties due to calculation limits.

Firstly, the contact surfaces in mobile micro-switches are generally of large dimension (of order of $20 \mu \mathrm{m}$ ) compared to the actual contacting region (of order of $500 \mathrm{~nm}$ ). The AFM technology isn't able to capture the total contact surface with a sampling interval limit of $1 \mathrm{~nm}$. The captured windows need to be reduced to limit the number of captured 3D data points. This process requires knowledge of the location of the contacting area, which is practically unfeasible.

Secondly, with a deterministic approach, the representation of real surface topography will demand a high volume of elements to include the micro-roughness. In three dimensional contact problems with elastic-plastic deformations, this involves long times of calculation and memory limitations [17].

The most probable ambiguity is the chosen resolution scale to scan and describe the rough surface topography. The resolution scale is given by the scan size and the number of registered data points by scanned line. A minimal scale has to be defined and the generated error has to be quantified if the finest roughness scales are neglected.

\section{INFLUENCE OF THE ROUGHNESS RESOLUTION SCALE IN MECHANICAL CONTACT ANALYSIS}

In order to find the appropriate sampling interval for our application, contact models for different contact surfaces of micro-switches were studied. We present in this section the methodology by considering one contact model built in two dimensions to reduce the number of contact elements in the numerical model. We use a partially idealized model which excludes the effect of contaminating film barriers and surface roughness of the contacting member that is pressed against the rough deformable body.

\section{A. Methodology using 2D finite element modeling}

The commercial software ANSYS is used to carry out our analyses. It is a standard tool used in industry for finite element analysis, historically well-known in mechanical and thermal problems. ANSYS is able to solve many contact problems (3D models, complex geometries, geometrical or material nonlinearities), with minimum effort from the user and has given good accuracy with reduced calculation times. 
An electroplated gold contact surface is captured by AFM on a resistive micro-switch. A 2D profile is then extracted along the discontinuous line illustrated in figure 2. A sampling interval limit of $1 \mathrm{~nm}$ is chosen because surface measurement with a smaller interval is ambiguous. The AFM data points are processed with Matlab in order to create a file with $\mathrm{z}(\mathrm{x}, \mathrm{y})$ coordinates compatible with ANSYS Parametric Design Language (APDL).

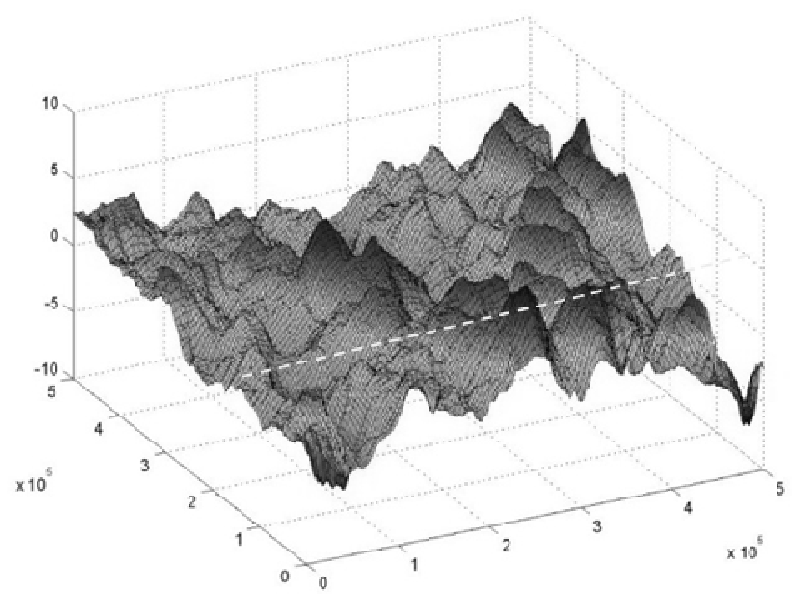

Fig. 2. AFM capture (processed with MATLAB) of an electroplated gold surface of size $500 \times 500 \mathrm{~nm}^{2}\left(\mathrm{R}_{\mathrm{a}}=2,07 \mathrm{~nm}, \mathrm{R}_{\mathrm{q}}=3,13 \mathrm{~nm}\right)$.

A plane stress contact problem is modeled by implementing the key-points imported from the Matlab file in the commercial code ANSYS. The surface is created by joining the successive key-points. In order to evaluate the impact of the finest details on asperities of the 2D profile, other contact models are built including key-points issued from smoothing of the initial profile. The smoothed profile is obtained by processing of the data points of the initial profile with a discretization step of $1 \mathrm{~nm}$ : the heights of discrete points belonging to the smoothed profile of degree $\mathrm{n}$ result from the height averaged on $\mathrm{n}$ consecutive data points of the initial profile. Figure 3 shows an example of the initial profile with two smoothed profiles at degrees 2 and 4 . In this way the finest successive levels of roughness are eliminated. The method for smoothing profiles is easy to carry out compared to the use of usual functions of interpolation . It avoids emergence of oscillations when a high degree is defined for the polynomial. Moreover this method proves to be consistent with the final way used to implement the discretized profile in finite elements code (cf column 2 page 6). The final calculation keeps indeed only one point out of $n$ consecutive ones and as a consequence the smoothed profile moves away from the initial profile.

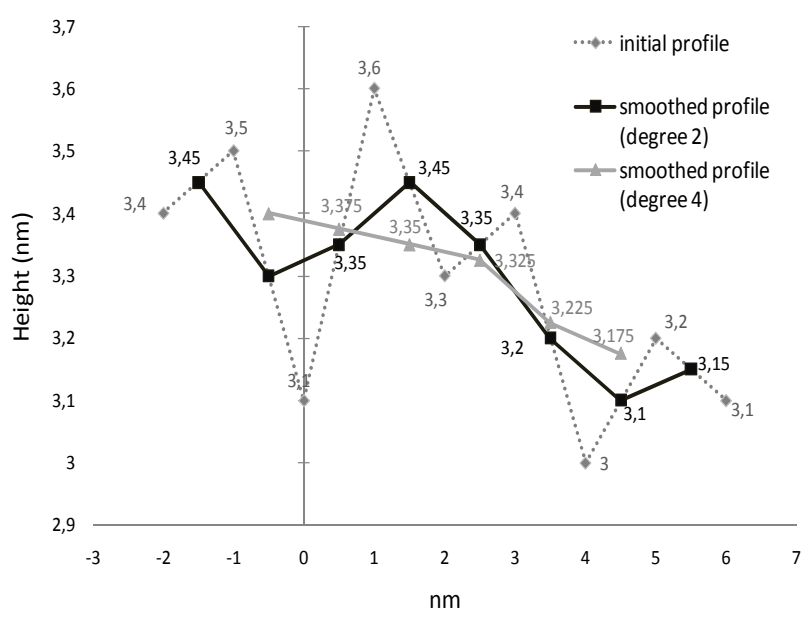

Fig. 3. Example of initial profile with smoothing at degrees 2 and 4.

Figure 4(a) illustrates the shapes of the different profiles issued from the initial profile extracted from the AFM capture. A zoom has been carried out in figure 4(b) in order to make the smoothed profiles of smaller degrees visible. The profiles are shifted for clarity.

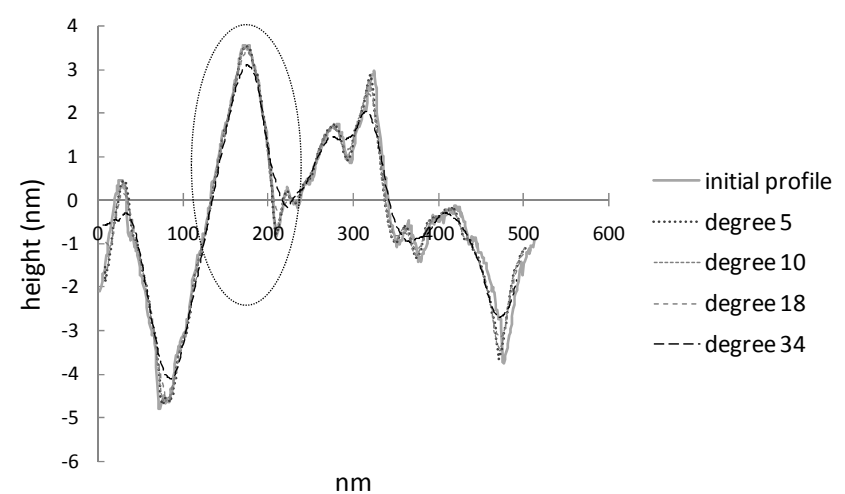

(a)

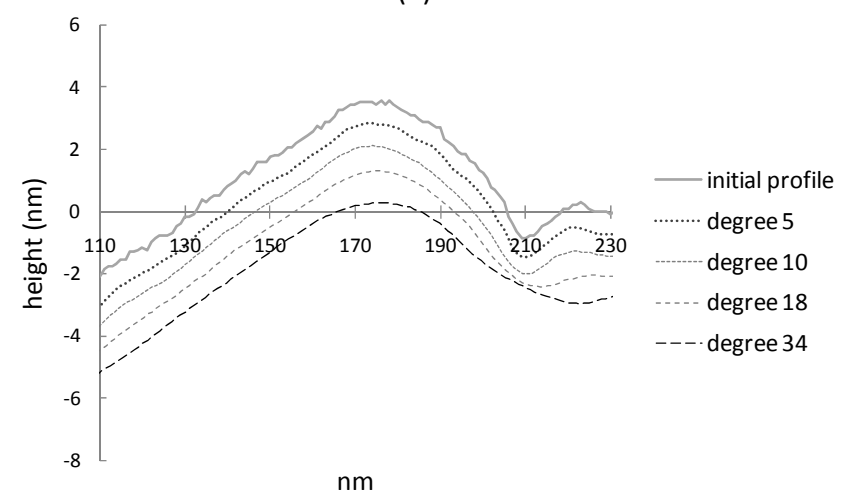

(b)

Fig. 4. Initial roughness profile and smoothed profiles at various degrees: (a) integral profiles, (b) zoom on moved profiles.

\section{B. Principle of the finite element contact analysis}

The finite element analysis is carried out using the structural static analysis of ANSYS11. The model is a rigid-to-flexible contact problem, for which one of the two contact surfaces is considered as rigid. This case is of great interest to reduce the number of elements in the model. The flexible surface material is defined with an equivalent 
Young's modulus [18]. The 2D contact model is thus made of a rigid indentor which is pressed against a $2 \mathrm{D}$ flexible block with the overlying rough surface on the top. We define for the flexible material elastic-plastic properties. Yield is introduced and the material behavior is described by a bilinear stress-strain curve. The initial slope of the curve is taken as the equivalent Young's modulus of the material. At the specified yield stress, the curve continues along the second slope defined by the tangential modulus of the material. We present the results obtained with gold material defined in table 1. Gold is often used in contact of micro-electromechanical systems due to its softness and low resistivity, which imply a low contact resistance, its relatively high melting point temperature for a soft material and its resistance to absorption of surface contaminants [3].

Table 1. Gold material properties

\begin{tabular}{lr}
\hline Young Modulus E & $80000 \mathrm{MPa}$ \\
Poisson Ratio $v$ & 0.42 \\
Yield Stress $\sigma_{0}$ & $300 \mathrm{MPa}$ \\
Tangent Modulus $\mathrm{E}_{\mathrm{t}}$ & $10000 \mathrm{MPa}$ \\
\hline
\end{tabular}

The deformable body is built using 2D structural solid elements PLANE183, defined by six nodes having two degrees of freedom at each node: translations in the $\mathrm{x}$ and $\mathrm{y}$ nodal directions. The element PLANE183 is well suited to modeling irregular meshes and has plasticity, stress stiffening, large deflection and large strain capabilities. Once the geometry is created, it is crucial to define the contact pairs, that is both surfaces that come into contact. The top surface is identified as the "contact" surface, whereas the created surface just touching and over the "contact" surface is defined as the "target". TARGE169 is used to represent the 'target' surface for the associated contact element CONTA172. The contact pair is associated with a set of constant like contact stiffness or penetration tolerance. Contact stiffness factor is very important in the contact analysis and its value has to be high enough with elastic-plastic materials to guarantee good accuracy of the results. Many simulations are thus performed with an increasing contact stiffness factor and the convergence of mechanical contact results is studied. Finally, a value of 50 is selected to run the calculations.

In order to restrict any movement of the base of the solid, the nodes of the base are rigidly constrained from moving. In our approach, a displacement is applied to the indentor causing it to move incrementally downward to flatten the surface asperities. A node is assumed to come into contact when its distance from the plane becomes zero.

The problem is thus displacement-controlled in order to make the contact convergence easier. This displacement is chosen in order that the contact force keeps the same value of $100 \mu \mathrm{N}$. The displacement is also close to $10 \mathrm{~nm}$.

To perform the contact analysis, the combined method based on penalty and Lagrangian methods and called the augmented Lagrangian method is chosen. The postprocessing generates the distribution of the contact pressure on the contact surface. An ANSYS command then gives directly the contact length for each 2D element.

\section{Results}

Once the contact analysis is carried out with ANSYS, the combined contact length is extracted from the postprocessing for each top surface profile. The results are presented in figure 5. A gap of $23 \%$ is observed between the contact lengths obtained with the initial profile and the smoothed profile at degree 2 , whereas this gap is reduced to $6 \%$ for the smoothed profiles at degrees 2 and 3 .

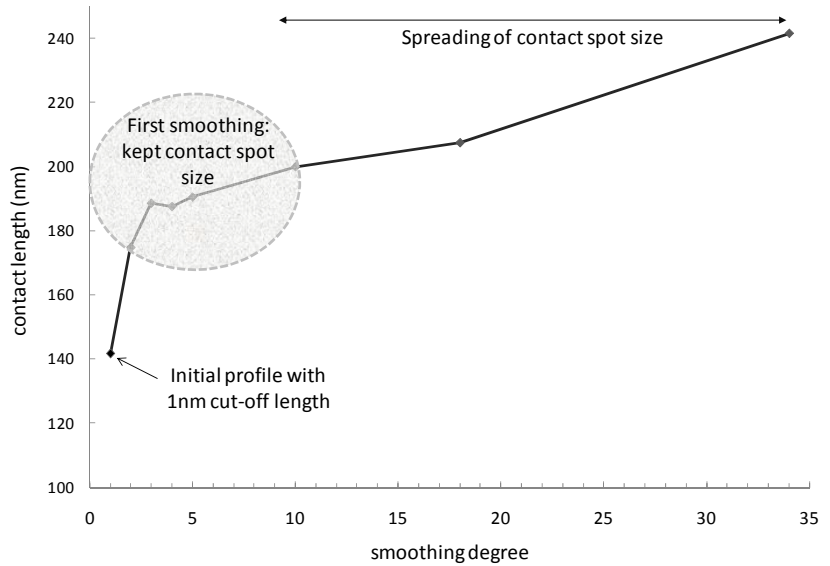

Fig. 5. Contact length as a function of the smoothing of rough surface profile.

To give an explanation of these results, some profiles after indentation are illustrated in figure 6. They highlight the emergence of six contact spots, but a zoom on a single contact spot underlines some discontinuities inside the spot issued from the initial profile. These discontinuities are insulating spots, that is of non contact. The smoothing leads to reduction of the number of insulating spots without significant loss on the original size of the contact spot. If the smoothing degree is increased beyond ten, the spot size becomes larger and spots can approach each other and merge. 


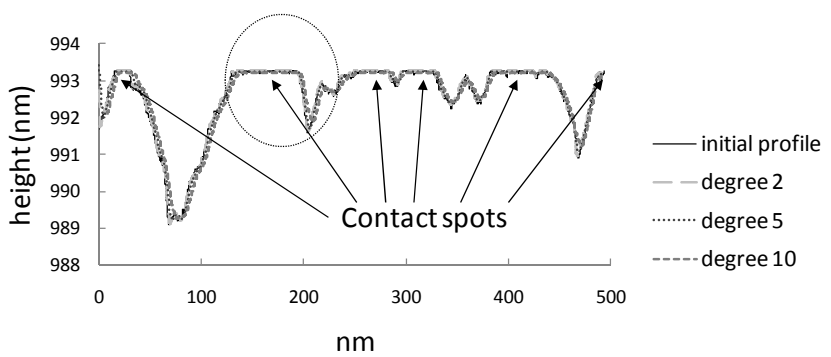

(a)

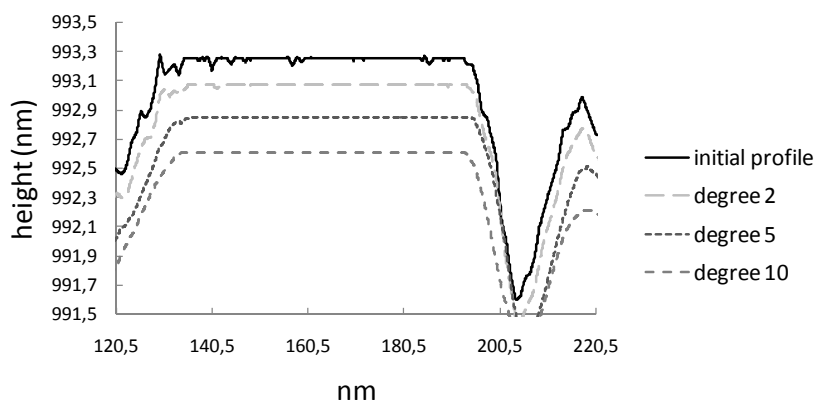

(b)

Fig. 6. Shape of the profiles after mechanical indentation: (a) integral profiles, (b) zoom on moved profiles.

The ANSYS mechanical contact analysis shows that roughness at a nanometer scale with asperities of height less than $1 \mathrm{~nm}$ doesn't wear off on larger asperities of radius close to $50 \mathrm{~nm}$ and under a contact pressure typically generated by micro-switches. The persistence of asperities in indentations during plastic bulk deformation has already been observed by Holm for originally annealed copper [19]. Gregori and Clarke have furthermore noticed that, after a large number of repeated contact events, local asperities are never completely flattened down [20]. It is important to evaluate the influence of the insulating spots on the flow of electrical current and the electrical contact resistance value and quantify the yield error by neglecting the discontinuities generated by the finest roughness scale.

IV. INFLUENCE OF THE ROUGHNESS RESOLUTION SCALE ON THE ELECTRICAL RESISTANCE

\section{A. Error evaluation}

In figure 6, the larger contact spots have a contact radius (a) close to the electron mean free path $l_{e}$ in gold $\left(l_{e} \sim 38 \mathrm{~nm}\right)$. The electron transport through the contact area is thus in part ballistic and in part diffusive or quasiballistic [21]. When ballistic electron transport dominates $\left(l_{e}>>a\right)$, the expression of Sharvin's resistance [22] is applied:

$$
R_{c B}=\frac{4 \rho K}{3 \pi a_{e f f}}
$$

where $\rho$ is the electric resistivity of contact material, and $K=\frac{l_{e}}{a_{e f f}}$ where $a_{e f f}$ is the effective radius of contact. When diffusive electron transport dominates $\left(\mathrm{l}_{\mathrm{e}}<<\mathrm{a}\right)$, the constriction resistance [19] is modeled analytically using:

$$
R_{c D}=\frac{\rho}{2 a}
$$

Then Wexler [23] interpolates between the ballistic and diffusive electron transport regions:

$$
R_{W}=R_{c B}+\Gamma(K) R_{c D}
$$

where $\Gamma(\mathrm{K})$ is a slowly varying Gamma function of unity order [24]. In this particular case, $K$ is close to the unit value and $\Gamma(\mathrm{K})$ is thus close to 0.5 .

Assuming a contact spot of radius $30 \mathrm{~nm}$ with 50 insulating spots inside of radius $1 \mathrm{~nm}$, the obtained discrepancy in the Sharvin resistance by neglecting the discontinuities is close to $6 \%$ according to the equation (1). The under-evaluation of the value reaches $12.5 \%$ with 100 insulating spots and $28.5 \%$ with 200 non contact spots.

Considering now the electron transport mode as diffusive, the current flow lines through the mechanical contact area can be modeled through finite element analyses. The commercial multi-physics tool COMSOL MULTIPHYSICS is used to carry out basic DC conductive simulations. An axisymmetric modeling of two gold cylinders forming an ideal circular contact of radius 30nm is considered and some insulating spots of $1 \mathrm{~nm}$ in edge length are successively added inside the spot (figures 7 and 8). Since the model is axisymmetric the insulating spots are in fact insulating rings. Fixed potential boundaries are applied at the circular base surface and at the circular top surface. The electrical resistance is calculated for each case between the two dotted sections that appear white in figure 7.

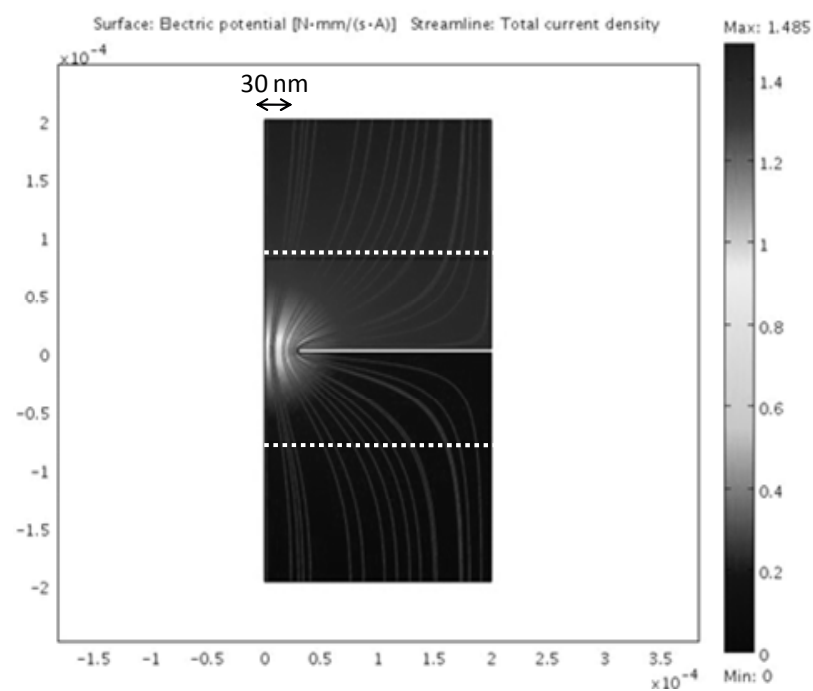

Fig. 7. Axisymmetric model built in COMSOL of a $30 \mathrm{~nm}$ radius gold contact spot without discontinuities inside the electrical constriction.

The electric resistance obtained by adding successively insulating spots is shown in figure 10. It is seen that the spots located at the contact interface periphery are responsible for deviation in the current lines and for resistance increase. Nevertheless with 24 contact spots, the maximal discrepancy reaches $4,4 \%$. These results agree with those of Holm [19]. 


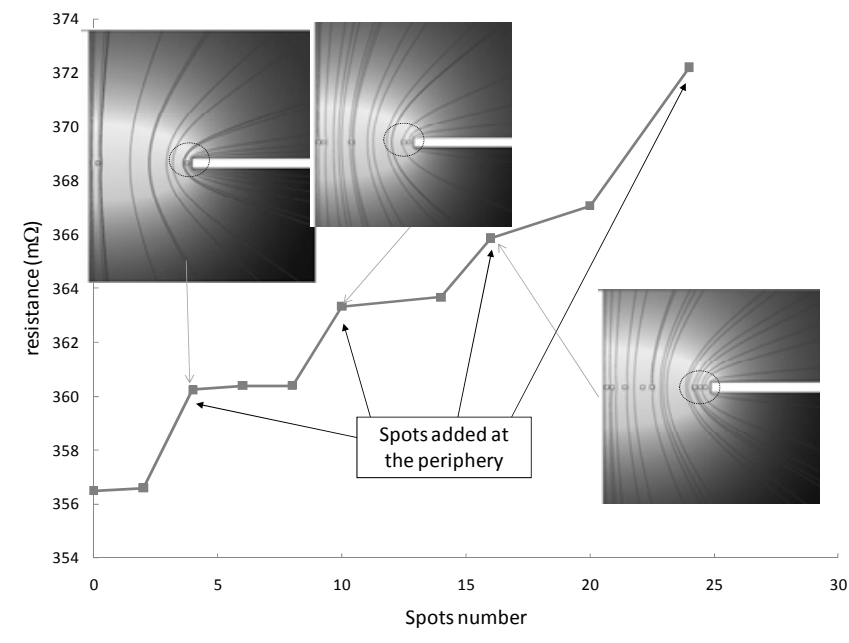

Fig. 8. Electric resistance versus the number of added insulating spots.

Combining the discrepancies obtained with the ballistic transport and the diffusive transport, the maximal error value is close to $5.4 \%$ with 50 insulating spots inside, $10 \%$ with about a hundred discontinuities and $21 \%$ with about 200 non contact spots.

The insulating contact spots have to be less than 100 inside the $30 \mathrm{~nm}$ radius contact spot to reduce the error to less than $10 \%$ when neglecting them in the electron conduction path.

\section{B. Choice of the sampling interval}

By considering the number of non contact spots rarely above 100, the choice of a smoothed profile, which keeps the contact spots size but suppresses the discontinuities inside, is well adapted for implementation in the numerical contact model and to obtain electrical contact results with a minor error. According to figure 5 a rough profile which has been smoothed to a degree close to 10 can thus be used to evaluate the electrical contact resistance since the contact spot size is maintained.

The drawback of the profile is the fact that it doesn't allow the number of degrees of freedom in the model to be reduced. We need in fact a surface profile with a reduced number of key points which define the rough profile and thus reduce the surface contact elements in the finite element model. The solution is to use discretized surface profiles in the 3D contact analysis of micro-switches. When the AFM captures the 3D surface topography, the sample size and the number of data points registered by the scanned line define the sampling interval of the measurement. Then, the data points can be processed using Matlab functions to generate filtered files with various discretization steps, equal or greater than the initial sampling interval. Another 2D contact analysis has thus been carried out with discretized profiles and compared to smoothed profiles. It has been checked that a surface profile with a discretization step of $10 \mathrm{~nm}$ instead of the initial $1 \mathrm{~nm}$ step yields essentially the same results as the surface profile smoothed at degree 10, that is smoothed with 10 data points or in this case with a $10 \mathrm{~nm}$ step. As it can be seen on figure 9, the contact spot size is kept.

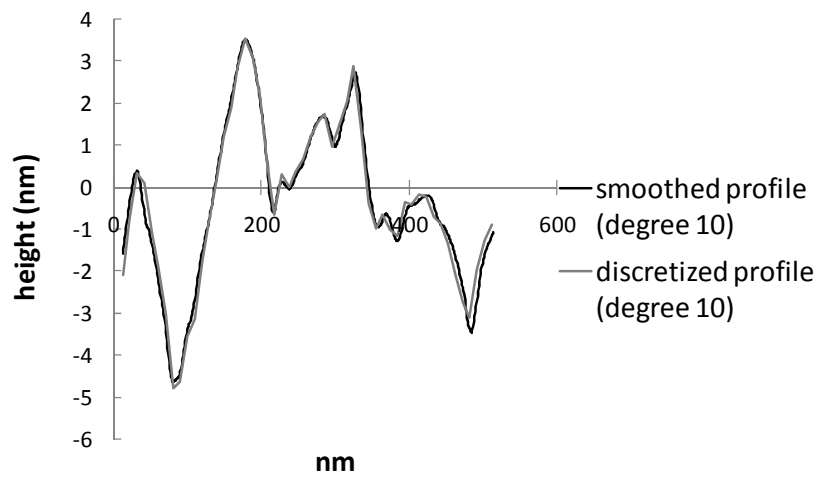

(a)

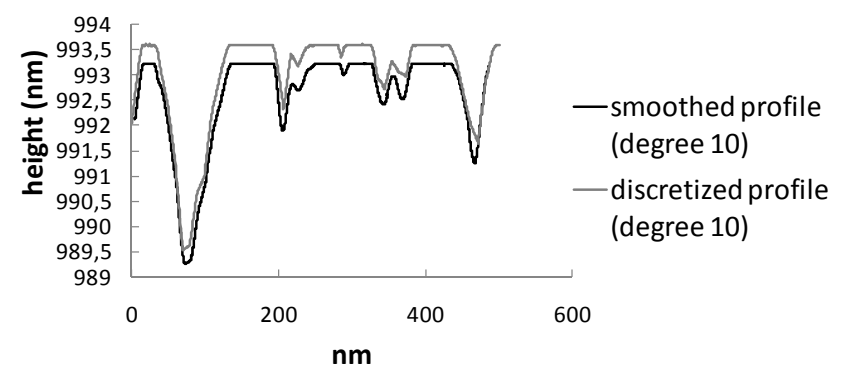

(b)

Fig. 9. Smoothed and discretized profiles before (a) and after mechanical indentation (b).

The rough profile discretized at $10 \mathrm{~nm}$ can thus be used to evaluate the electrical contact resistance and reduce the number of surface contact elements in a 3D simulation by a factor of 100 compared with a rough profile with a $1 \mathrm{~nm}$ cut-off length.

\section{IMPLEMENTATION ON MEMS TEST STRUCTURES}

\section{A. Choosing a test case}

To test the method to perform contact simulation of actual surfaces, the method is implemented on test structures fabricated in the LETI laboratories. The testing devices are composed of a gold bridge suspended above the contact line. A $6 \mu \mathrm{m}$ bump is patterned on the line. The bumps consist of silica glass overlaid with $1 \mu \mathrm{m}$ thickness plated gold. The material contact is thus $A u-t o-A u$.

\section{B. Methodology of contact analysis}

Contact models are built between the rough deformable contact bump with a resolution step close to $10 \mathrm{~nm}$ and a plane and smooth deformable indenter. Contact material is gold as detailed previously in table 1.

The AFM is used to capture three dimensional data points of contact bumps. The AFM instrument stores 512 data points per scanned line and so the resolution step is equal to $11.7 \mathrm{~nm}$.

Then using Matlab functions we convert the closed surfaces from a stereo-lithographic format to an ASCII file. We can choose to keep the resolution step from the 3D capture or to filter the files in order to obtain larger steps of roughness definition. 
An example of geometry of a finite element mesh for a rough contact bump is illustrated in figure 10 with a discretization step equal to $140 \mathrm{~nm}$.

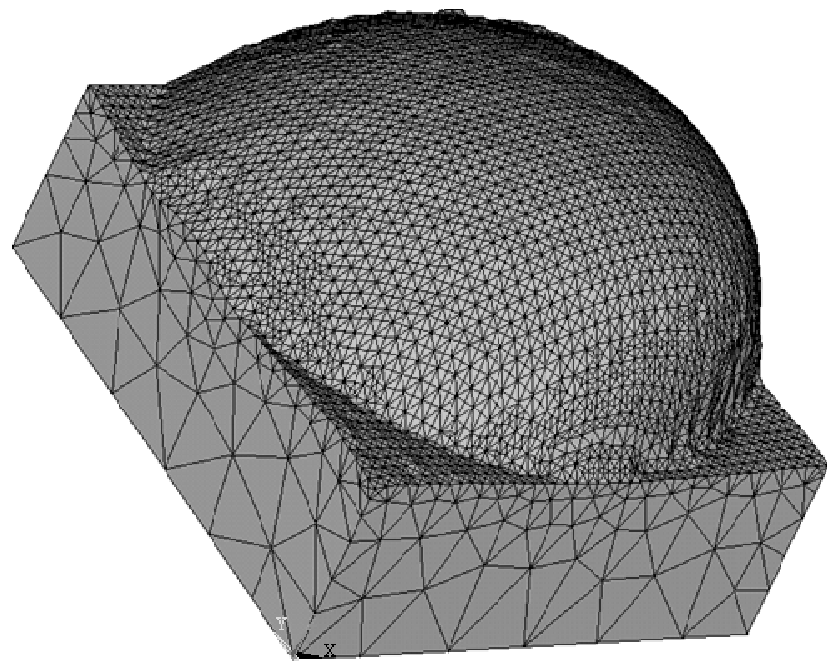

Fig. 10. Geometry of finite element mesh in rough contact bump with the overlying surface generated from data points imported from ASCII file $\left(\mathrm{R}_{\mathrm{a}}=15,2 \mathrm{~nm}, \mathrm{R}_{\max }=96 \mathrm{~nm}\right)$.

A first series of finite element analyses is run with the resolution step of the line roughness close to $140 \mathrm{~nm}$ to detect the effective contact region. The contact force is applied to the top plane surface of the membrane. Figure 11 shows the contact penetration on the contact surface of the bump illustrated in figure 13 for an applied force equal to $100 \mu \mathrm{N}$. The locations where the penetration is non null are denoted as contact regions.

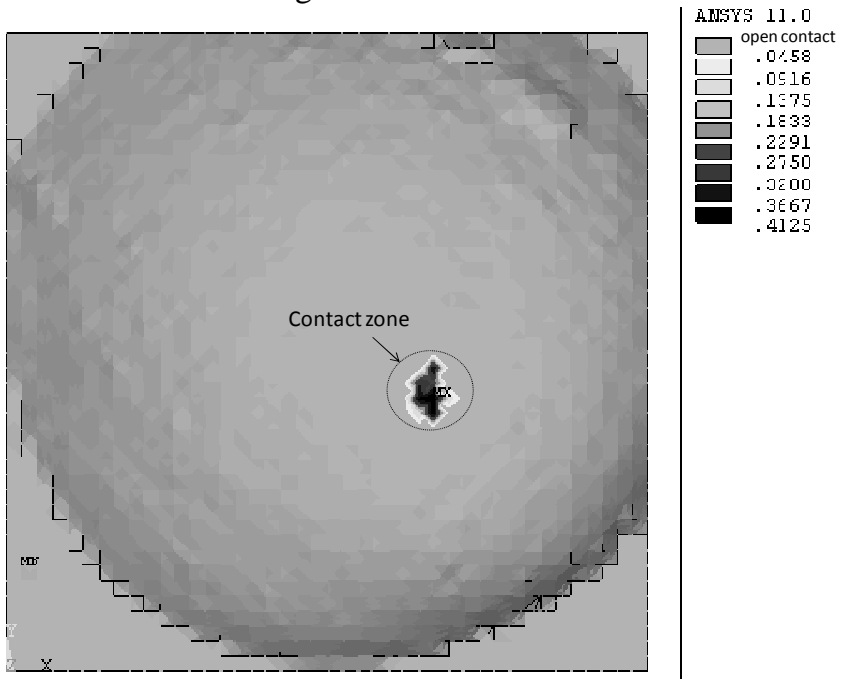

Fig. 11. Contact penetration (nm) on the rough contact bump.

One single contact spot appears on the contact surface with an equivalent radius of $189 \mathrm{~nm}$. This value is larger than the electron mean free path of gold $\left(l_{e} \sim 38 \mathrm{~nm}\right)$. In this case, the electrical contact resistance is due to a diffusive transport of electron through the contact area.

The constriction resistance is modeled analytically using Holm's expression (2) to be evaluated at $58.4 \mathrm{~m} \Omega$.

The following step consists in reducing the roughness resolution step close to $10 \mathrm{~nm}$ to get accurate results. However shortening the discretization step leads to a large amount of elements in the contact model. As the effective contact region has been located, the problem can be addressed by defining locally the surface topography around the effective detected contact region. Our objective is to use the smallest step of resolution $(11.7 \mathrm{~nm})$. This length is very small compared to the sample dimensions $\left(6 \times 6 \mu m^{2}\right)$ and thus we reduce its dimensions to $1.5 \mathrm{x}$ $1.5 \mu \mathrm{m}^{2}$, by checking with a larger resolution step that these reductions don't affect the mechanical contact results.

It can be noticed that a finite element calculation with a sampling interval of $1 \mathrm{~nm}$ limits the model dimensions to 150x150 $\mathrm{nm}^{2}$ for memory limitations reasons. Such simulation involves some discrepancies in bulk deformations and is thus not validated. In addition, dimensions of AFM capture with a sampling interval of $1 \mathrm{~nm}$ are often limited to $500 \times 500 \mathrm{~nm}^{2}$. These difficulties require many $3 \mathrm{D}$ captures on the contact bump and can be tedious to perform when the contact region is not located in advance.

\section{Results and discussion}

Figure 12 illustrates the contact pressure distribution obtained with a step of $11.7 \mathrm{~nm}$. The $11.7 \mathrm{~nm}$ discretization interval allows a reduction of the equivalent contact radius of $36 \%$. Many contact spots emerges on the apparent contact surface and are due to the appearance of asperities on larger asperities when a resolution step close to $10 \mathrm{~nm}$ is chosen. This case highlights the importance if finer details of roughness are implemented in the contact model on the mechanical contact results.

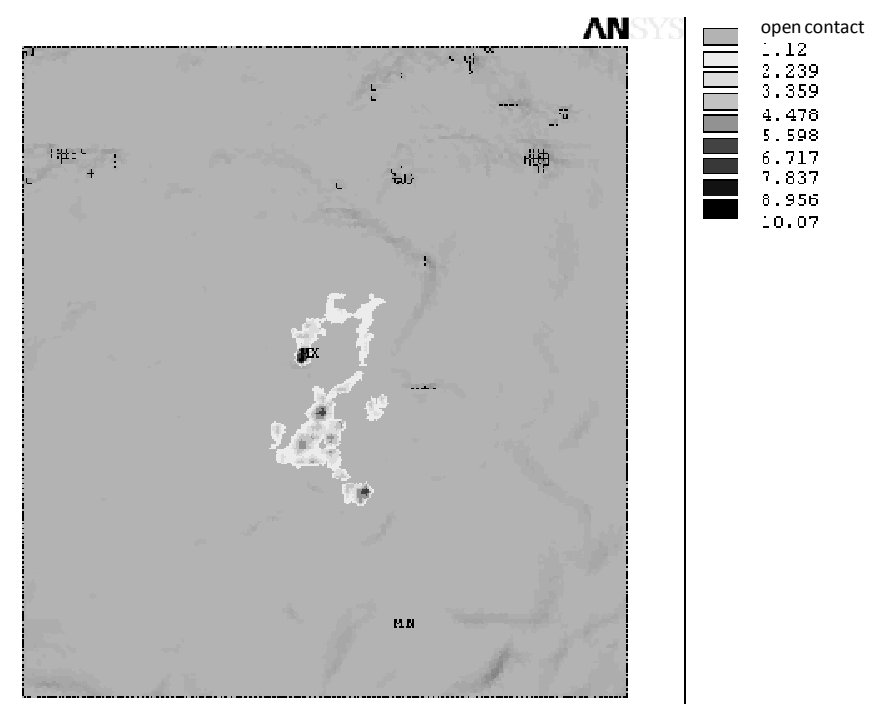

Fig. 12. Distribution of the contact pressure (GPa) on the top of the rough contact bump.

Greenwood derived a formula for the constriction resistance of a set of circular spots when the electrodes communicate via the spots with no interface film between them. 


$$
R_{G 1}=\frac{\rho}{2 \sum a_{i}}+\frac{\rho}{\pi} \cdot \frac{\sum \sum \frac{a_{i} a_{j}}{d_{i j}}}{\left(\sum a_{i}\right)^{2}}
$$

with $a_{i}$ the radius of the spot $i, d_{i j}$ the distance between the centers of the spots $i$ and $j$.

We apply this formula to deduce the electrical contact resistance generated with a resolution step of $11.7 \mathrm{~nm}$.

The $11.7 \mathrm{~nm}$ allows a resistance increase of only $11.5 \%$ due to the fact that many contact spots appear on the bump surface. The calculated contact resistance is close to $65 \mathrm{~m} \Omega$. If we consider an under-evaluation of the contact resistance due to the choice of the $12 \mathrm{~nm}$ discretization step, the value reaches $71.5 \mathrm{~m} \Omega$.

Contact analyses have been performed on three other rough bumps and have demonstrated that the simulated contact resistances with a $12 \mathrm{~nm}$ discretization step are found in the $63-72 \mathrm{~m} \Omega$ range.

It is difficult to compare these numerical results with experimental values since many assumptions are made on our contact models. The roughness on the beam and the contaminant films are notably not taken into account.

However stable contact resistance values between 80 and $200 \mathrm{~m} \Omega$ are measured and listed in the literature with an applied contact force close to $100 \mu \mathrm{N}$ when choosing gold as contact material [3-5]. These experimental values are promising for continuing our research effort on the improvement of the contact modeling based on actual surface topography.

\section{CONCLUSIONS}

The study of metal-to-metal contact becomes crucial to guarantee high performances of DC contact switches with actuation voltages. When estimating the resistance of contact structures, it must be taken into consideration the importance of including fine scale details of the surface roughness contributing to the limitation of current flow through a cluster of contact spots and increase of the total contact resistance. The atomic force microscope is used to capture 3D data points of surface topography with a sampling interval decreasing to $1 \mathrm{~nm}$. This work can be long and tedious when the contact region $(10 \mu \mathrm{m}$ radius bump) is large and the effective contacting zone (200 nm radius region) unknown. In addition, the implementation of the roughness with a resolution step of $1 \mathrm{~nm}$ implies a large number of contact elements and so of calculation time. The definition of elastic-plastic materials in the model implies even an overloading, preventing the calculations to succeed. The need of reducing the number of degrees of freedom in the contact model in order to overcome the technical difficulties leads to the study of the influence of the smallest roughness level on the electrical current conduction. Contact analyses showed that a sampling interval of $10 \mathrm{~nm}$ is sufficient if we tolerate an error of ten percent in the electrical contact results.
The method has been implemented on test structures but can't be actually validated since many assumptions are made. The surface roughness of the beam has been neglected, the contaminating film barriers have not been included in the model and the thermal effects or material transport due to the flow of electric current aren't taken into account. However the results are promising with respect to the experimental values found in the literature. Other efforts are required in the research on the development of a contact model based on actual surface topography with low forces. The method will be validated with experimental measurement on MEMS test structures for many contact materials. The tool is of great interest for investigating the impact of materials, roughness, technological process of the RF MEMS contact.

\section{ACKNOWLEDGMENT}

This study was supported by the ANR (Agence Nationale de la Recherche) in the frame of the FAME project and by the French Industry Ministry in the frame of SPRINT project. The authors would like to thank Christina Villeneuve of LAAS-CNRS for the AFM measurements and Christel Dieppedale of CEA-LETI for the fabrication of gold-to-gold test structures.

\section{REFERENCES}

[1] H. R. Shea, "Reliability of MEMS for Space Applications", in proc. Of SPIE Reliability, Packaging, Testing and Characterization of MEMS/MOEMS, vol.6111, 2006.

[2] G. M. Rebeiz, and J. B. Muldavin, "RF MEMS Switches and Switch Circuits”, IEEE Microwave Magazine, Dec. 2001.

[3] G. Rebeiz, "RF MEMS Theory, Design and Technology" New Jersey: Wiley, 2003, pp. 192-199.

[4] J. Schimkat, "Contact materials for microrelays", Proc. IEEE MEMS '98, Heidelberg Germany, pp. 333-337, 1998.

[5] D. Hyman and M. Mehregany, "Contact physics of gold microcontacts for MEMS switches”, IEEE Trans. on components and packaging technology, vol. 22, No3, pp. 357-364, 1999.

[6] F.P. Bowden and D. Tabor, "Friction: An Introduction to Tribology”, Anchor Press/Doubleday, Garden City, 1973.

[7] B.N.J. Persson, "Elastic contact between randomly rough surfaces”, Phys. Rev. Lett., vol. 87, pp. 1-4, 2001.

[8] J.F. Archard, "Elastic deformation and the laws of friction", Proc. R. Soc. Lond. A, vol. 243, pp. 190-205, 1957.

[9] M. Ciavarella, G. Demelio, J.R. Barber, J.H. Jang, "Linear elastic contact of the Weierstrass profile”, Proc. Roy. Soc. Lond., Ser. A, vol. 456, pp.387-405, 2000.

[10] E. Ciulli, L.A. Ferreira, G. Pugliese, S.M.O. Tavares, "Rough contacts between actual engineering surfaces Part.I. Simple models for roughness description”, Wear, vol. 264, pp.1105-1115, 2008

[11] C. Vallet, D. Lasseux, P. Sainsot, H. Zahouani, « Real versus synthetized fractal surfaces: contact mechanics and transport properties », Tribology International, vol. 42, pp.250-259, 2009

[12] J.A. Greenwood, J.B.P Williamson, "Contact of nominally flat surfaces”, Proc. R. Soc. Lond. A, vol. 295, pp. 300-319, 1966.

[13] P. Sahoo, N. Ghosh, "Finite Element contact analysis of fractal surfaces”, J. Phys. D: Appl. Phys., vol. 40, pp. 4245-4252, 2007.

[14] S. Lee, H. Cho, Y.H. Jang, "Multiscale electrical contact resistance in clustered contact distribution” J. phys. D: Appl. Phys, vol. 42, pp. $1-7,2009$

[15] B. Bhushan, «Methodology for roughness measurement and contact analysis for optimization of interface roughness ", IEEE transactions on magnetics, vol. 32, N³, pp. 1819-1825, may 1996. 
[16] B. Bhushan, G.S. Blackman, "Atomic force microscopy of magnetic rigid disks and sliders and its applications to tribology", ASME J. tribilogy, vol. 113, pp. 452-457, 1991.

[17] B. Bhushan, "Contact mechanics of rough surfaces in tribology: multiple asperity contact” Tribology Letters, vol. 4 pp. 1-35, 1998

[18] K.L. Johnson, « Contact mechanics », Cambridge University Press, Cambridge, 1985.

[19] R. Holm, "Electric contacts: Theory and Applications" $4^{\text {th }}$ edition, Berlin, Germany: Springer-Verlag, 1967.

[20] G. Gregori, D.R. Clarke, "The interrelation between adhesion, contact creep, and roughness on the life of gold contacts in radiofrequency microswitches” J. Appl. Phys. Vol. 100, 2006

[21] R.A. Coutu, J.R. Reid, R. Cortez, R.E. Strawser and P. Kladitis, "Microswitches with Sputtered Au, AuPd, Au-on-AuPt, and AuPtCu Alloy Electric Contacts”, IEEE transactions on Components and Packaging Technologies, vol. 29, No.2, June 2006

[22] Y. V. Sharvin, Sov. Phys. JETP 21, 655, 1965.

[23] G. Wexler, "the size effect and the nonlocal Boltzmann transport equation in orifice and disk geometry”, in Proc. Phys. Soc., Vol. 89, pp. 927-941,1966.

[24] A. Mikrajuddin, F. Shi, H. Kim, K. Okuyama, "Size-dependant electrical constriction resistance for contacts of arbitrary size: from Sharvin to Holm limits”, in Proc. Mater. Sci. Sem., Vol. 2, pp. 321327, 1999

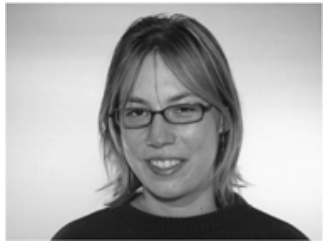

Fabienne Pennec received the B.S degree in mechanical engineering from national engineering institute in mechanics and microtechnologies, Besançon, France, in 2005 and the M.S. and Ph.D. degrees in micro and nanosystems from the Toulouse University and LAAS-CNRS in 2005 and 2009, respectively.

David Peyrou received Ph.D. and M.S degrees in mechanical and microsystems from the Toulouse University and LAAS-CNRS in 2006 and 2003, respectively. He received Agrégation in Mechanical 2002, highest-level teaching diploma in France, prepared as student from Ecole Normale Supérieure, Cachan (thereafter mentioned as ENS) and the B.S degree in mechanical engineering from national engineering institute in mechanics and microtechnologies, Besançon, France, in 2001. He was postdoctoral Fellow and Research Assistant in 2008, at the Laboratory for Analysis and Architecture of Systems (LAAS), one of the laboratories of CNRS, the French National Center for Scientific Research.

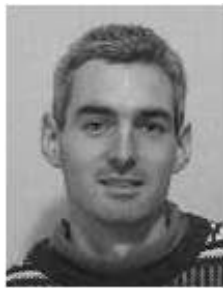

Dimitri Leray received the Ph.D. degree in Mechanical Engineering from Toulouse University in 2002. His current works focus on simulation and mechanical characterization of microstructures, as well as simulation and electromechanical characterization of microswitch contact.

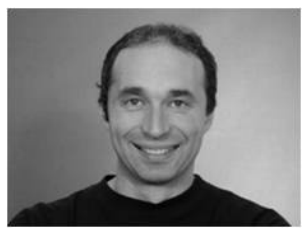

Patrick Pons received received Ph.D degree in electronics from Toulouse University (France), in 1990. Since 1991 he is a researcher in the CNRS-LAAS (National Scientific Research Center - Laboratory of Analysis and Architecture of Systems) in Toulouse. His research interests are focused in microtechnology and microsensors. In 1995, he started in the lab the study on microtechnology for microwave applications. Now he manages the development of this technology for high frequency Microsystems and develops also pressure sensors for specific applications. In 2005 he started research in a new field coupling sensors and RF for the development of passive wireless sensors.

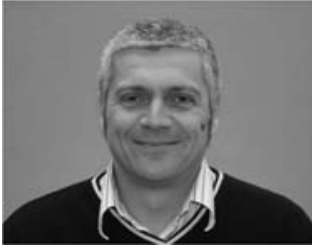

Robert Plana obtained its $\mathrm{PhD}$ in 1993 at LAAS-CNRS and Paul Sabatier University on the Noise modelling and characterization of Advanced Microwave devices (HEMT, PHEMT and HBT) that includes the reliability. In 1993, as associate professor at LAAS-CNRS, he has started a new research area concerning the investigation of millimeterwave capabilities of Silicon based technologies. In 1995, he has started a new project concerning the improvement of the passives on silicon through the use of MEMS technologies. In 1999, he has been involved with SiGe Semi-conductor in Ottawa where he was working on the low power and low noise integrated circuits for RF applications. In the same year, he has received a special award from CNRS for his works on Silicon based technologies for millimeterwave communications. In 2000, he has been professor at Paul Sabatier University and Institut Universitaire de France and he has started a research team at LAAS-CNRS in the field of Micro and Nanosystem for RF and millimeterwave communications. Its main interests are on the technology, design, modelling, test, characterization and reliability of RF MEMS for low noise and high power millimeterwave applications and the development of the MEMS IC concept for smart microsystem. He has built a network of excellence in Europe in this field “AMICOM" regrouping 25 research groups. He has authored and co-authored more than 225 international journals and conferences. In 2004, he has been appointed as Deputy Director of the Information and Communication Department at the CNRS Headquarter. From January 2005 to January 2006, he has been appointed director of the Information and Communication Department at CNRS. Since 2006, he is heading a research group at LAAS-CNRS in the field of Micro and Nanosystem for wireless communications. Since 2008, he also joined the "French research Agency" where he is the project officer of the National Nanotechnology Initiative.

Frédéric Courtade is currently staff engineer with CNES, the French Space Agency, He is Expert on EEE component technologies \& processes and Technical Officer for Research \& Technology studies on micro nano technologies. 